\title{
Protective Effects of Plathymenia reticulata and Connarus favosus Aqueous Extracts against Cadmium- and Mercury-Induced Toxicities
}

\author{
Kewin Gombeau ${ }^{1,2}$, Ricardo Bezerra de Oliveira $^{3}$, Sandra Layse Ferreira Sarrazin ${ }^{3}$, \\ Rosa Helena Veras Mourão ${ }^{3}$ and Jean-Paul Bourdineaud ${ }^{1,4}$ \\ ${ }^{1}$ University of Bordeaux, CNRS, UMR 5805, EPOC, Arcachon Marine Station, 33120 Arcachon, France \\ ${ }^{2}$ Present address: University of Bordeaux, CNRS, UMR5095 CNRS, Institute for Cellular Biochemistry and Genetics, \\ 1 rue Camille Saint Saëns, CS 61390, 33077 Bordeaux cedex, France \\ ${ }^{3}$ Federal University of Western Pará - UFOPA, PPGRNA, LABBEX, Tapajós Campus, Rua Vera Paz s/n, Bairro Salé, CEP, \\ 68040-050, Caranazal, 88040-060 Santarém, Pará, Brazil \\ ${ }^{4}$ Present address: University of Bordeaux, CNRS, UMR MFP 5234, European Institute of Chemistry and Biology, 2 rue \\ Robert Escarpit, 33607 Pessac, France
}

\begin{abstract}
The extracts of Plathymenia reticulata and Connarus favosus are widely used in the folk medicine. The potential protective effects of these extracts have been evaluated against cadmium in the yeast Saccharomyces cerevisiae, and against mercurial contamination in zebrafish Danio rerio. In yeast, both extracts efficiently protected the $\Delta y c f 1$ mutant strain exposed to cadmium chloride restoring the growth, the expression of stress-response genes and decreasing the level of oxidative stress. In zebrafish, the supplementation of methylmercury-contaminated diet with both plant extracts similarly protected fish through the suppression of the methylmercury-induced lipid peroxidation, decrease of acetylcholinesterase activity, and restoring the expression levels of stress-response genes. This study particularly demonstrates the protective potential of both aqueous extracts against methylmercury, and could represent an interesting alternative for the Amazonian fish-eating communities to cope with the impact of chronic exposure to contaminated diets.
\end{abstract}

Key words: Plant extract, Methylmercury, Cadmium, Saccharomyces cerevisiae, Danio rerio, Oxidative stress

\section{INTRODUCTION}

Since the dawn of civilization, medicinal plants have been used to alleviate diseases and afflictions. Furthermore, they are still currently widely used as the sole therapeutic option for many isolated communities in Brazil, where ethnobotanical surveys have confirmed the presence of several species endowed with medicinal proper-

Correspondence to: Jean-Paul Bourdineaud, University of Bordeaux, CNRS, UMR MFP 5234, European Institute of Chemistry and Biology, 2 rue Robert Escarpit, 33607 Pessac, France

E-mail: jean-paul.bourdineaud@u-bordeaux.fr

This is an Open-Access article distributed under the terms of the Creative Commons Attribution Non-Commercial License (http:// creativecommons.org/licenses/by-nc/3.0) which permits unrestricted non-commercial use, distribution, and reproduction in any medium, provided the original work is properly cited. ties (1-4).

Recently, two aqueous extracts were chemically characterized. The first, from Plathymenia reticulata Bentham (Fabaceae), is rich in condensed tannins and phenolic compounds (5), while the second, from Connarus favosus Planch. (Connaraceae) is rich in condensed tannins, phenolic compounds, flavonoids, and catechins (6). These extracts are widely used in folk medicine, particularly to treat snakebites (7). Their efficacy as snakebite anti-venoms has been confirmed in vitro through the inhibition of the hemorrhagic activity of Bothrops atrox venom $(5,6)$. However, several other medicinal properties need to be validated. $P$. reticulata is known by the vernacular name candeia or vinhático (8) and is used to treat inflammation, pain, and kidney troubles (9).

Humans, especially Amerindian and riverine populations in the Amazonian basin are exposed to hazardous 
mercurial contamination mainly through fish consumption, and the consequences are decreased motor performance and visual impairment (10). The use of elemental liquid mercury $\left(\mathrm{Hg}^{0}\right)$ by clandestine gold miners and the natural richness of the Amazonian soils in inorganic $\mathrm{Hg}$ has led to the production of methylmercury $(\mathrm{MeHg})$ in the aquatic environment through the activity of anaerobic bacteria. MeHg biomagnifies up the food chain and carnivorous fish eventually become highly contaminated (10). A diet rich in fruits would decrease the mercury uptake through fish consumption in human communities in the Brazilian Amazon $(11,12)$. Therefore, it is fairly possible that the use of traditional plant extracts may play a protective role against mercurial exposure.

The goal of this study was to evaluate the potential protective effects of both extracts against metal-induced toxicity, following direct exposure to cadmium chloride $\left(\mathrm{CdCl}_{2}\right)$ in yeast. In addition, we also investigated the protective effects against chronic contamination of zebrafish with mercury-contaminated food mimicking the level of exposure of carnivorous fish in the Amazonian basin (10). At the end of the 60-day exposure period, the mercury accumulation levels of the brains, livers, and skeletal muscles of the fish were measured as well as the lipid peroxidation, acetylcholinesterase (AChE) activity, and expression of a selected subset of genes. Indeed, $\mathrm{MeHg}$ is known to inhibit AChE activity in mammalian species and fish, disrupting the mitochondrial respiratory chain, thereby increasing the reactive oxygen species levels, altering calcium homeostasis, and increasing lipid peroxidation (13-18).

\section{MATERIALS AND METHODS}

Aqueous plant extract preparation. Two tree species of Amazonia have been used to extract useful tannins: the bark of $P$. reticulata Bentham (Fabaceae), and the inner bark of C. favosus Planch. (Connaraceae). Samples of the inner bark of $C$. favosus were collected in São Pedro community, Santarém, Pará, Brazil $\left(2^{\circ} 32^{\prime} 8.9^{\prime \prime} \mathrm{S}\right.$ and $\left.54^{\circ} 54^{\prime} 23.9^{\prime \prime} \mathrm{W}\right)$. Samples of $P$. reticulata bark were collected close to the community of Cucurunã, Santarém, Pará, Brazil ( $2^{\circ} 26^{\prime} 53.9^{\prime \prime} \mathrm{S}$ and $\left.54^{\circ} 47^{\prime} 5^{\prime \prime} \mathrm{W}\right)$. Water extraction of these vegetal materials and determination of their major chemical class of compounds was performed as previously described for the aqueous extracts of $P$. reticulata (5) and C. favosus (6).

Yeast culture and selection of working extract doses in zebrafish feed. The potential protective effects of $P$. reticulata and $C$. favosus extracts were first investigated in the $\Delta y c f 1$ mutant yeast Saccharomyces cerevisiae challenged with toxic doses of $\mathrm{CdCl}_{2}(20$ or $40 \mu \mathrm{M}$, known to trigger the onset of an oxidative stress and DNA damage) using two concentrations of both extracts ( 500 or 1,000 $\mathrm{mg} / \mathrm{L}$ ) and monitoring the biomass growth. This mutant strain is hypersensitive to drugs and cadmium because it lacks a vacuolar ATP-binding cassette transporter, which allows vacuolar transfer and accumulation of glutathione (GSH)-conjugated drugs and cadmium (19). Additionally, changes in the expression patterns of genes related to the oxidative and mutagenic stress response (GSH1, KAR2, paired related homeobox $1(P R X I)$, and RNA, ribosomal $45 \mathrm{~S}$ cluster $3[R N R 3]$ ) were analyzed using quantitative real-time polymerase chain reaction (qRT-PCR). These genes were selected because they are well known to be upregulated when the oxidative and mutagenic status of the cell is increased $(20,21)$. This allowed the selection of an effective dose of the extract to be incorporated in the zebrafish feed. The $\Delta y c f 1 S$. cerevisiae strain was cultivated as described in the Additional file. The biomass growth was assessed by quantifying the number of copies of the GSH1 gene as described in the Additional file. The qRT-PCR analysis of the yeast mentioned above gene expression was carried out as described in the Additional file (Supplementary Table 1).

Zebrafish culture and experimental diet preparation. The experimental diets for the zebrafish (supplemented, contaminated, or both) were prepared by mixing artificial fish food (Dr. Bassleer Biofish, Telgte, The Netherlands) with reagent-grade alcohol (95\% ethanol, Prolabo, France) and $0.5 \%$ (5 mg extract per $\mathrm{g}$ of food) of the plant aqueous extract or dissolved methylmercury chloride (reference 33368, Sigma-Aldrich), or both as previously described (22). Wild-type adult zebrafish were purchased from a commercial company (Exomarc, Lormont, France), and acclimatized for 7 days at $23^{\circ} \mathrm{C}$ in a large tank filled with dechlorinated water (body weight: $0.79 \pm 0.03 \mathrm{~g}$, wet weight; standard length: $3.33 \pm 0.07 \mathrm{~cm}, n=6$ ). Twenty adult fish were randomly placed in six different plastic tanks with $20 \mathrm{~L}$ of dechlorinated water, continuously aerated by bubbling air, and were daily given a quantity of food representing $5 \%$ of their body weight (b.w.). Control fish were fed with uncontaminated food (Dr. Bassleer Biofish, Telgte). Under the exposure conditions designated "MeHg," the fish were fed contaminated food containing $13.5 \mu \mathrm{g} \mathrm{MeHg} / \mathrm{g}$ dry weight (d.w.). Under the assumption that $100 \%$ of the ingested $\mathrm{MeHg}$ is absorbed, the zebrafish were exposed to $675 \mu \mathrm{g} \mathrm{Hg} \cdot$ day $^{-1} \cdot \mathrm{kg}$ b.w ${ }^{-1}$. This concentration of $\mathrm{MeHg}$ was chosen because of its environmental relevance to the mercury content of fish eaten by other piscivorous fish and based on our previous studies showing that it decreased mitochondrial respiration and ATP synthesis rates in skeletal muscles (23). In addition, it disorganized the mitochondrial cristae structure of the skeletal muscles (24), induced a strong $\mathrm{MeHg}$ demethylation process in the liver (22), and impaired the optical tectum integrity, with decreased cell density (25). In the 
exposure condition hereafter called "P. reticulata" or " $C$. favosus," the fish were fed with control food supplemented with $0.5 \%(\mathrm{w} / \mathrm{w})$ of either the P. reticulata or C. favosus extract at an average dose of ingested extract equal to 250 $\mathrm{mg} \cdot \mathrm{kg}^{-1} \cdot$ day b.w. ${ }^{-1}$. This concentration was chosen based on the results observed in yeast exposed to $\mathrm{CdCl}_{2}$ and treated with the extracts. Indeed, at a concentration of 500 $\mathrm{mg} / \mathrm{L}$, both extracts exerted a potent beneficial effect on yeast by increasing the survival and decreasing oxidative stress after a $\mathrm{CdCl}_{2}$ challenge (see the following results). Similarly, under the exposure conditions hereafter designated "P. reticulata $+\mathrm{MeHg}$ " and "C. favosus $+\mathrm{MeHg}$ ", the food was spiked with $13.5 \mu \mathrm{g} \mathrm{MeHg} / \mathrm{g}$ d.w. and supplemented with $0.5 \%$ of either the P. reticulata or C. favosus extract. To minimize fish contamination by the water, half of the water volume from each tank was changed every 2 days, and tank bottoms were cleaned every day to eliminate fish feces, and food remains.

After 60 days of exposure to the different diets, the fish were collected, euthanized within seconds by immersion in melting ice in agreement with the ethical guidelines displayed and used by the NIH intramural research program. Then, the fish were dissected to assess the mercury accumulation, mercurial neurotoxicity, lipid peroxidation, and gene expression.

Mercury quantification. The mercury concentrations in the food (13.6 $\pm 0.90 \mu \mathrm{g} \mathrm{Hg} / \mathrm{g}$ d.w., $n=3)$ and fish tissues were determined using flameless atomic absorption spectrometry, and carried out automatically after thermal decomposition at $750^{\circ} \mathrm{C}$ under an oxygen flow (AMA 254, Altec, Prague, Czech Republic) with a detection limit of $0.01 \mathrm{ng} \mathrm{Hg}$. The precision (\% of relative standard deviation) was $10 \%$, and the accuracy of the analytical methodology was determined for each set of measurements by analyzing the certified reference material (CRM) in lobster hepatopancreas, TORT-2. CRM determined values: $0.27 \pm 0.03$ ng $\mathrm{Hg} / \mathrm{mg}$ dry weight (mean $\pm \mathrm{SD}, n=120$ measurements); CRM recommended value: $0.27 \pm 0.06 \mathrm{ng} \mathrm{Hg} / \mathrm{mg}$ d.w.).

Lipid peroxidation assessment. The level of lipid peroxidation has been investigated using the thiobarbituric acid reactive substances (TBARS) assay, which allows the quantification of oxidative stress by measuring the peroxidative damage to lipids that occurs with free radical generation. These damages to lipids are associated with the production of malondialdehyde (MDA), which reacts with thiobarbituric acid (TBA) under specific temperature and acidic condition, generating a chromogen measurable spectrofluorometrically. The TBARS assay was conducted according to a previously published protocol (26) using the 1,1,3,3-tetramethoxypropane (TMP, ref: 108383, SigmaAldrich, St Louis, MO, USA) as an external standard. The total protein content was measured using the Bradford method based on the ability of Coomassie blue to bind proteins. The levels of lipid peroxidation are expressed as nanomoles (nmol) of TBARS/mg of proteins.

Measurement of AChE activity. The activity of AChE, a neurotoxicity biomarker, was assessed in the brains and skeletal muscles of fish using the most common method (27) using microplate spectrometry. This assay is based on acetylthiocholine iodide cleavage by AChE and the subsequent reaction of thiocholine with the chromogenic disulfide 5,5-dithiobis 2-nitrobenzoic acid. The reaction was run for 5 minutes following the addition of the substrate, and the optical density (OD) was measured every $13 \mathrm{sec}-$ onds. Then, the AChE activity was calculated using the molar extinction coefficient of the 5-thio-2-nitrobenzoic acid $\left(13,600 \mathrm{~L} \cdot \mathrm{mol}^{-1} \cdot \mathrm{cm}^{-1}\right)$ and expressed as nanomoles (nmol) per hydrolyzed substrate milligram protein per minute ( $\mathrm{mg}$ protein $/ \mathrm{min}$ ). The total protein content was measured using the Bradford method based on the binding of Coomassie blue to proteins. We used the Bio-Rad protein assay dye reagent concentrate (ref: 5000006), and bovine serum albumin (BSA, ref: A2153, Sigma-Aldrich) as the standards.

Total RNAs extraction and qRT-PCR analysis. Total RNA was extracted from five whole brains, livers, and skeletal muscles using the Absolute RNA RT-PCR Miniprep kit (Stratagene, Agilent Technologies, Les Ulis, France) according to the manufacturer's protocol. To eliminate the maximum lipids and proteins, a phenol-chloroform-isoamylic alcohol $(25: 24: 1)$ extraction step was added just after the homogenization step. Following the extractions, first-strand cDNA was synthesized from total the RNA using the Stratascript First-Strand Synthesis System (Stratagene, Agilent) according to the manufacturer's instructions. The cDNA samples were kept at $-20^{\circ} \mathrm{C}$ until used in the real-time PCR reactions, which were performed using a thermocycler (MX3000P qPCR System, Stratagene, Agilent) using Brilliant III Ultra-Fast SYBR Green QPCR Master Mix (Stratagene, Agilent Technologies, Santa Clara, CA, USA) according to the manufacturer's recommendations. The parameters set to perform the qRT-PCR reactions are provided in the Additional file. The primers used in the qRT-PCR reactions are given in Supplementary Table 2.

Statistical analyses. All statistical analyses were performed using three to five biological replicates per condition. First, the normality and homogeneity of the variance were tested using the Shapiro-Wilks test and Levene test respectively $(\alpha=0.05)$. If normality and homoscedasticity were confirmed, a Student's $t$-test was performed $(\alpha=$ $0.05)$. Otherwise, the Student's $t$-test was replaced by the Mann-Whitney test $(\alpha=0.05)$. 
Table 1. Effect of natural extracts on the growth of the yeast $\Delta y c f 1$ mutant exposed to cadmium (in \% of the cadmium-untreated and extract-free control yeast growth)

\begin{tabular}{|c|c|c|c|c|c|}
\hline \multirow{2}{*}{$\begin{array}{l}\mathrm{CdCl}_{2} \\
(\mu \mathrm{M})\end{array}$} & \multirow{2}{*}{$\begin{array}{l}\text { Without } \\
\text { extract }\end{array}$} & \multicolumn{2}{|c|}{ P. reticulata } & \multicolumn{2}{|c|}{ C. favosus } \\
\hline & & $500 \mathrm{mg} / \mathrm{L}$ & $1000 \mathrm{mg} / \mathrm{L}$ & $500 \mathrm{mg} / \mathrm{L}$ & $1000 \mathrm{mg} / \mathrm{L}$ \\
\hline 0 & $100 \pm 6^{\mathrm{a}}$ & $99 \pm 2$ & $99 \pm 4$ & $108 \pm 8$ & $100 \pm 7$ \\
\hline 20 & $46 \pm 3$ & $81 \pm 7^{\S}$ & $91 \pm 5^{\S}$ & $92 \pm 4^{\S}$ & $122 \pm 19^{\S}$ \\
\hline 40 & $36 \pm 7$ & $52 \pm 5^{\S}$ & $55 \pm 5^{\S}$ & $47 \pm 5$ & $48 \pm 24$ \\
\hline
\end{tabular}

The yeast biomass has been assessed through the quantification of the number of copies of the GSH1 gene by qPCR. Values are means \pm SD $(n=3)$.

'The number of GSH1 gene copies for the control without extract was equal to $(1.8 \pm 0.1) \times 10^{5}$ in the assay, corresponding to a Ct equal to $21.81 \pm 0.08(n=3)$.

${ }^{\varsigma}$ This symbol indicates significant differences between growth values in the presence of natural extract compared to the extract-free control, for the same concentration of cadmium, as assessed by the Mann-Whitney test $(p<0.05)$.

\section{RESULTS}

Protection conferred by $P$. reticulata and $C$. favosus extracts to yeast against the effects of cadmium. To evaluate the protective properties of the natural extracts in the yeast mutant strain $\Delta y c f l$ exposed to $\mathrm{CdCl}_{2}$, we monitored the growth (Table 1) and measured the expression levels of oxidative and mutagenic stress-response genes (Table 2).

Both natural extracts alleviated the cadmium-induced decreased growth (Table 1). Indeed, after exposure to $20 \mu \mathrm{M} \mathrm{CdCl}$, which resulted in a $54 \%$ inhibition of growth, treatments with 500 and $1,000 \mathrm{mg} / \mathrm{L}$ of the $P$. reticulata extract significantly induced a growth increase of 35 and $45 \%$ compared to the cadmium-exposed condition, respectively (Table 1). Similar effects were observed after exposure to $40 \mu \mathrm{M} \mathrm{CdCl}$ and treatment with 500 and $1,000 \mathrm{mg} / \mathrm{L} P$. reticulata extract, which significantly increased the growth by 16 and $19 \%$, respectively compared to the cadmium-exposed condition (Table 1). Treatment with the $C$. favosus extract also reduced the impact of $\mathrm{CdCl}_{2}$ exposure on growth only at a concentration of $20 \mu \mathrm{M} \mathrm{CdCl}$. Treatments with 500 and $1,000 \mathrm{mg} / \mathrm{L} C$. favosus extract significantly increased the growth by 46 and $76 \%$, respectively compared with the $20 \mu \mathrm{M}$ cadmiumexposed condition (Table 1).

Additionally, we evaluated the protective effects of both natural extracts on the level of expression of four oxidative and mutagenic stress-response genes (Table 2). The exposure of the yeast mutant $\Delta y c f l$ to both $\mathrm{CdCl}_{2}$ concentrations significantly and affected the expression levels of the studied genes, in a concentration-dependent manner (Table 2). Indeed, exposure to $20 \mu \mathrm{M} \mathrm{CdCl}_{2}$ induced a 3and 2-fold increase in the expression level of the KAR2 and the $P R X 1$ genes compared to the control condition, respectively (Table 2). However, exposure to the highest concentration of $\mathrm{CdCl}_{2}$ induced a significant over-expression of the four studied genes compared to the control levels, with 2.6-, 3.4-, 2.8-, and 5.5-fold increased levels of
Table 2. Effect of natural extracts on the expression of yeast genes involved in the resistance to oxidative stress in the $\Delta y c f 1$ mutant exposed to cadmium

\begin{tabular}{|c|c|c|c|c|}
\hline \multirow{2}{*}{$\begin{array}{c}\mathrm{CdCl}_{2} \\
(\mu \mathrm{M})\end{array}$} & \multicolumn{4}{|c|}{ Genes } \\
\hline & GSH1 & KAR2 & PRX1 & RNR3 \\
\hline 0 & $25 \pm 15$ & $60 \pm 34$ & $46 \pm 28$ & $10 \pm 8$ \\
\hline 20 & $39 \pm 5$ & $182 \pm 28^{*}$ & $95 \pm 3 *$ & $30 \pm 6$ \\
\hline 40 & $66 \pm 12^{*}$ & $204 \pm 35^{*}$ & $130 \pm 15^{*}$ & $55 \pm 21^{*}$ \\
\hline \multicolumn{5}{|c|}{$\begin{array}{l}\text { Plathymenia reticulata bark extract } \\
5500 \mathrm{mg} / \mathrm{L}\end{array}$} \\
\hline 0 & $7.5 \pm 1.5$ & $11 \pm 6$ & $3 \pm 2$ & $0.6 \pm 0.3$ \\
\hline 20 & $2.3 \pm 0.7^{\S}$ & $6 \pm 2^{\S}$ & $3.8 \pm 1.5^{\S}$ & $0.45 \pm 0.3^{\S}$ \\
\hline 40 & $2.7 \pm 1.7^{\S}$ & $15 \pm 3^{\S}$ & $2.2 \pm 0.8^{\S}$ & $0.3 \pm 0.1^{\S}$ \\
\hline \multicolumn{5}{|c|}{$1000 \mathrm{mg} / \mathrm{L}$} \\
\hline 0 & $1.5 \pm 0.1$ & $1.5 \pm 0.7$ & $2.2 \pm 0.8$ & $1.5 \pm 0.8$ \\
\hline 20 & $4.5 \pm 3.5^{\S}$ & $2.2 \pm 0.7^{\S}$ & $3.8 \pm 2.2^{\S}$ & $0.5 \pm 0.1^{\S}$ \\
\hline 40 & $1.2 \pm 0.2^{\S}$ & $5.0 \pm 0.1^{\S}$ & $4.5 \pm 2.2^{\S}$ & $1.0 \pm 0.6^{\S}$ \\
\hline \multicolumn{5}{|c|}{$\begin{array}{l}\text { Connarus favosus inner bark extract } \\
\qquad 500 \mathrm{mg} / \mathrm{L}\end{array}$} \\
\hline 0 & $6 \pm 2$ & $7 \pm 2$ & $5 \pm 2$ & $8 \pm 2$ \\
\hline 20 & $21 \pm 7$ & $33 \pm 8^{\S}$ & $22 \pm 6^{\S}$ & $22 \pm 9$ \\
\hline 40 & $17 \pm 6^{\S}$ & $21 \pm 9^{\S}$ & $15 \pm 7^{\S}$ & $11 \pm 6$ \\
\hline \multicolumn{5}{|c|}{$1000 \mathrm{mg} / \mathrm{L}$} \\
\hline 0 & $14 \pm 13$ & $8 \pm 3$ & $12 \pm 8$ & $5 \pm 0,4$ \\
\hline 20 & $7 \pm 1^{\S}$ & $14 \pm 5^{\S}$ & $8 \pm 2^{\S}$ & $8 \pm 1^{\S}$ \\
\hline 40 & $0.5 \pm 0.3^{\S}$ & $1.7 \pm 0.9^{\S}$ & $0.6 \pm 0.3^{\S}$ & $0.7 \pm 0.2^{*, \S}$ \\
\hline
\end{tabular}

The gene of the $18 \mathrm{~S}$ ribosomal RNA were used as reference. All values were multiplied by 1000 to facilitate reading. Values are means \pm SEM $(n=3)$.

*This symbol indicates significant differences between the expression values in the presence of cadmium compared to cadmiumuntreated control, for the same concentration of natural extract as assessed by the Mann-Whitney test $(p<0.05)$.

${ }^{5}$ This symbol indicates significant differences between the expression values in the presence of natural extract compared to the extract-free control, for the same concentration of cadmium, as assessed by the Mann-Whitney test $(p<0.05)$.

expression of $G S H 1, K A R 2, P R X 1$, and $R N R 3$ genes, respectively (Table 2 ), indicating the expected onset of an 
oxidative stress and damage to DNA.

The treatment of cadmium-exposed yeast mutant $\Delta y c f 1$ with both natural extracts suppressed the cadmium-induced over-expression of the oxidant and mutagenic stress-response genes, and the protective effects were much prominent with the $P$. reticulata treatment that with $C$. favosus. Additionally, whatever the exposure concentration of $\mathrm{CdCl}_{2}$, both concentrations of each extract maintained the expression level of these genes similar to those of the cadmiumuntreated control, except for C. favosus extract at a concentration of $1,000 \mathrm{mg} / \mathrm{L}$ during a challenge with $40 \mu \mathrm{M}$ of $\mathrm{CdCl}_{2}$ (Table 2).

Hg accumulation in zebrafish organs. After 60 days of exposure, a significant level of mercury accumulation was detected in all the tissues of fish exposed to the different $\mathrm{MeHg}$-spiked diets as compared to the control condition (Fig. 1). Indeed, in the organs of fish from the $\mathrm{MeHg}$ condition, the concentrations measured in the brain, the liver, and the skeletal muscle were respectively $215-, 255-$ and 64-fold higher than those measured in control fish (Supplementary Table 3).

In the tissues of fish fed food containing $\mathrm{MeHg}$ and supplemented with the $P$. reticulata extract, a significant accu- mulation was observed as compared to control fish (236-, 128-, and 64-fold higher in the brain, the liver and the skeletal muscle respectively Supplementary Table 3). In these fish, the levels of accumulated mercury in the brain and the skeletal muscle were similar to those measured in the $\mathrm{MeHg}$ exposure condition (Fig. 1). However, this combined exposure induced a significant 2-fold lower level of mercury accumulated in the liver compared to those measured under the MeHg condition (Supplementary Table 3).

Additionally, the levels measured in the tissues of fish fed with food containing $\mathrm{MeHg}$ and the C. favosus extract were significantly higher than those measured in the control fish (230-, 206-, and 58-fold higher in the brain, the liver, and the skeletal muscle respectively, Supplementary Table 3), and similar to those measured in fish fed $\mathrm{MeHg}$ contaminated food (Fig. 1).

Suppression of $\mathbf{M e H g}$-generated oxidative stress. To evaluate the antioxidant properties of the plant extracts against mercurial toxicity, the levels of lipid peroxidation were measured using the TBARS assay in the brain, liver, and skeletal muscle of the fish. The data obtained following a 60-day exposure to MeHg-contaminated food (Fig. 2) confirmed that the mercurial pro-oxidant property with

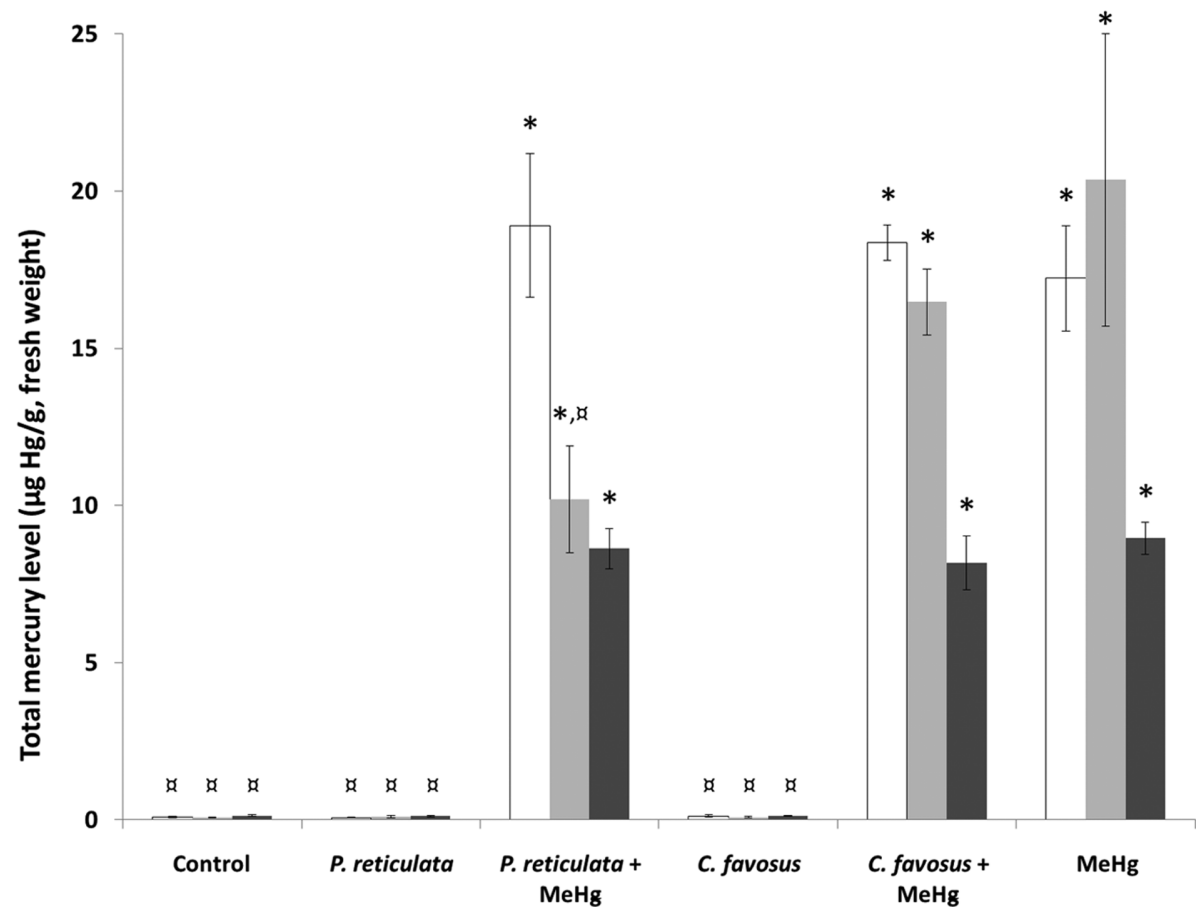

Fig. 1. Total $\mathrm{Hg}$ levels measured in the brain (white bars), the liver (light grey bars) and the skeletal muscle (dark grey bars) of zebrafish exposed to different diets during 60 days. Values are presented as mean \pm standard deviation $(\mathrm{SD})(n=3)$. ${ }^{*}$ Significantly different from the control group ( $\left.{ }^{*} p<0.05\right)$. ${ }^{\text {"}}$ Statistical difference as compared to the MeHg exposure condition $\left({ }^{\mathrm{a}} p<0.05\right)$. In the " $P$. reticulata" and "C. favosus" exposure conditions fish were fed with control food supplemented with $0.5 \%$ of either the $P$. reticulata or the $C$. favosus extract. In the exposure conditions "P. reticulata $+\mathrm{MeHg}$ " and "C. favosus $+\mathrm{MeHg}$ ", the food was spiked with $13.5 \mu \mathrm{g}$ $\mathrm{MeHg} / \mathrm{g}$ d.w. and supplemented with $0.5 \%$ of either the $P$. reticulata or the $C$. favosus extract. In the exposure condition called "MeHg", fish were fed with contaminated food containing $13.5 \mu \mathrm{g} \mathrm{MeHg} / \mathrm{g}$ d.w. 


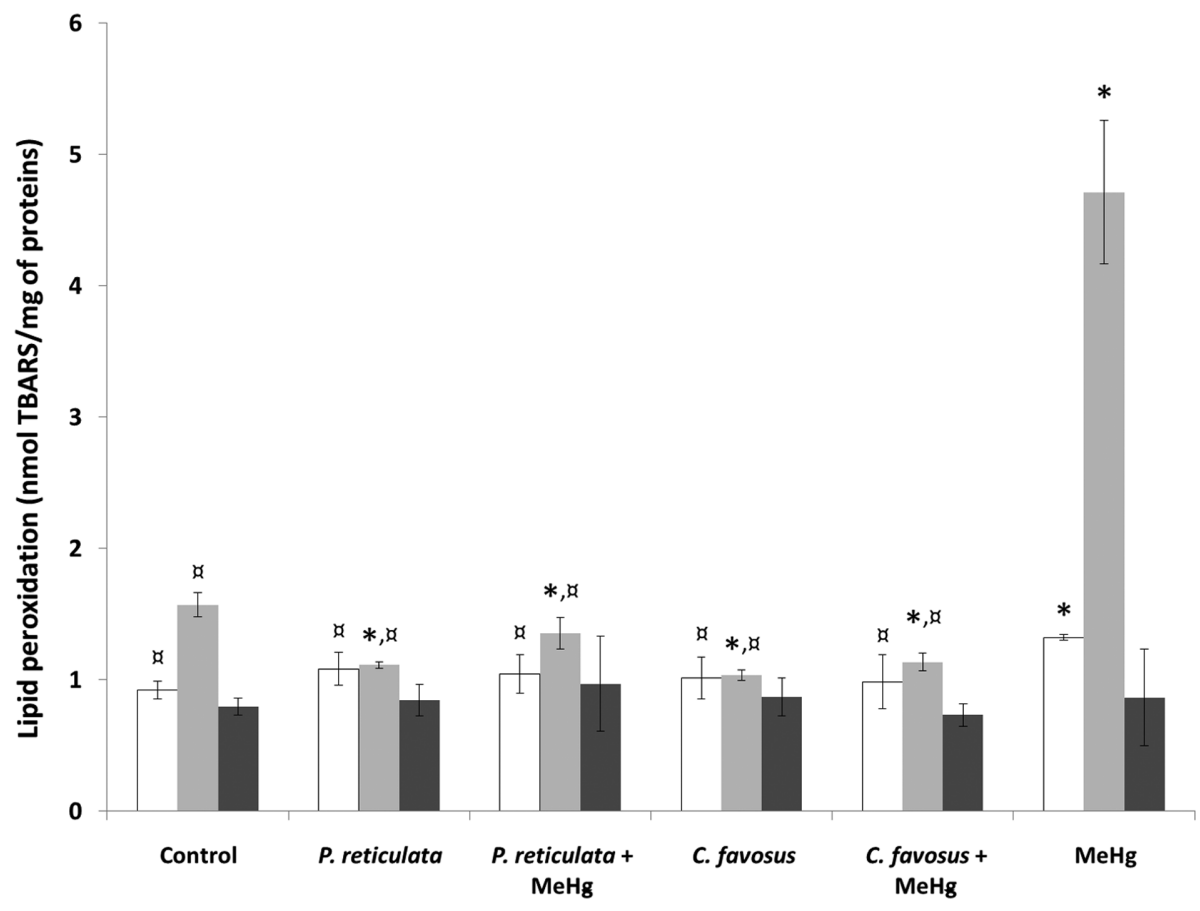

Fig. 2. Assessment of the lipid peroxidation in the brain (white bars), the liver (light grey bars) and the skeletal muscle (dark grey bars) of zebrafish exposed to different diets during 60 days. Values are presented as mean \pm SD $(n=3)$. *Significantly different from the control group ( ${ }^{*} p<0.05$ ). "Statistical difference as compared to the MeHg exposure condition ( $\left.p<0.05\right)$. In the "P. reticulata" and "C. favosus" exposure conditions fish were fed with control food supplemented with $0.5 \%$ of either the $P$. reticulata or the $C$. favosus extract. In the exposure conditions "P. reticulata + MeHg" and "C. favosus + MeHg", the food was spiked with $13.5 \mu \mathrm{geHg} / \mathrm{g}$ d.w. and supplemented with $0.5 \%$ of either the $P$. reticulata or the C. favosus extract. In the exposure condition called "MeHg", fish were fed with contaminated food containing $13.5 \mu \mathrm{g} \mathrm{MeHg} / \mathrm{g}$ d.w.

a significant $50 \%$ increase in the lipid peroxidation level in the brain and a 3 -fold higher level of TBARS in the liver of fish compared to the controls (Supplementary Table 4).

The antioxidant property of the plant extracts was assessed by the supplementation of the control food by $0.5 \%$ of either the $P$. reticulata extract or the $C$. favosus extract. Indeed, the levels measured in the livers of these fish pointed out a significantly reduced basal level of lipid peroxidation by 30 and $34 \%$ compared to the controls after treatment with $P$. reticulata and $C$. favosus extracts, respectively (Supplementary Table 4 ). After spiking the vegetal extract-supplemented food with $\mathrm{MeHg}$, not only did both $P$. reticulata and $C$. favosus extracts neutralize the oxidative stress generated by the $\mathrm{MeHg}$ uptake with observed 3-fold decreased levels of MDA in liver, but also maintained an MDA level below 14 and $27 \%$ of that of the control fish liver, respectively (Fig. 2). In the brain of these fish, the MeHg-induced lipid peroxidation was suppressed by both extracts. The TBARS levels measured in this organ were significantly lower than those observed in the MeHg-exposed fish were (20 and 25\% decreased values after treatment with food containing $\mathrm{MeHg}$ and $P$. reticulata or C. favosus extract, respectively; Supplementary Table 4), and similar to those measured in the controls.
Neuroprotective potential of plant extracts. The mercurial neurotoxicity and neuroprotective potential of both plant extracts have been evaluated by the assessment of the specific initial activity of the AChE in the brain and skeletal muscle of fish fed with the different diets (Fig. 3). The results obtained following supplementation of the control food with $0.5 \%$ the $P$. reticulata extract (Fig. 3) pointed out a significant increase of the AChE activity, with a 26 and $34 \%$ higher activity both in the brain and the skeletal muscle of fish as compared to controls (Supplementary Table 5). In contrast, food supplementation with $0.5 \%$ of the C. favosus extract (Fig. 3) also stimulated the AChE activity, but this was only significant in the skeletal muscle with a $40 \%$ increase compared with the controls (Supplementary Table 5).

Following a 60-day exposure to $\mathrm{MeHg}$-contaminated food (Fig. 3), the mercurial neurotoxic effects were highlighted by a significant $14 \%$ and $36 \%$ decrease of the AChE activity in the brain and skeletal muscle of fish as compared to the control condition (Supplementary Table 5).

The neuroprotective potential of the plant extracts was highlighted by their suppression of the mercurial neurotoxicity (suppression of the inhibition of the AChE activity). On the one hand, supplementation of the $\mathrm{MeHg}$ - 


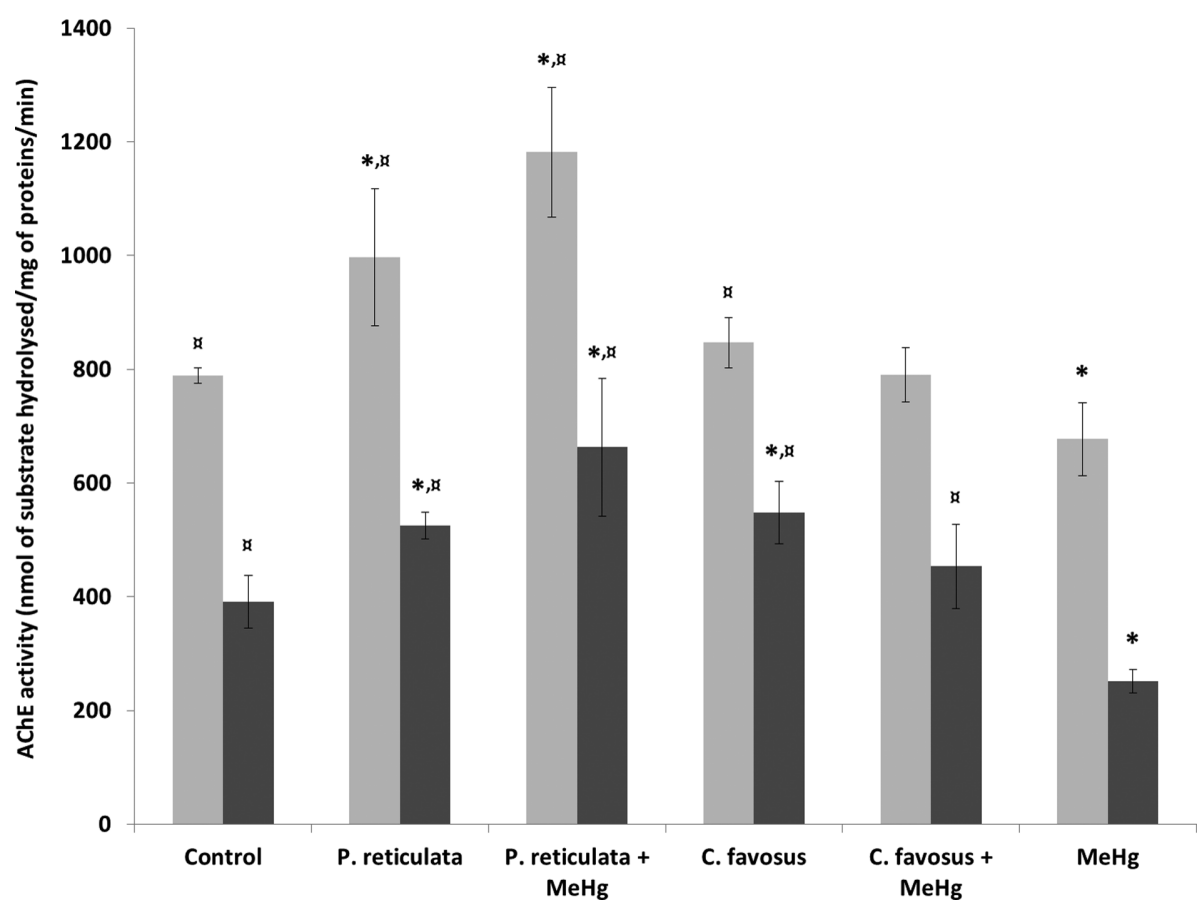

Fig. 3. Quantification of the specific initial activity of the AChE in the brain (light grey bras), and the skeletal muscle (dark grey bars) of zebrafish exposed to different diets during 60 days. Values are presented as mean \pm standard error of mean (SEM) ( $n=3$ ). *Significantly different from the control group $\left({ }^{*} p<0.05\right)$. "Statistical difference as compared to the MeHg exposure condition (" $p<0.05)$. In the "P. reticulata" and "C. favosus" exposure conditions fish were fed with control food supplemented with $0.5 \%$ of either the $P$. reticulata or the $C$. favosus extract. In the exposure conditions "P. reticulata $+M e H g$ " and " $C$. favosus + MeHg", the food was spiked with $13.5 \mu \mathrm{g} \mathrm{MeHg} / \mathrm{g}$ d.w. and supplemented with $0.5 \%$ of either the P. reticulata or the C. favosus extract. In the exposure condition called "MeHg", fish were fed with contaminated food containing $13.5 \mu \mathrm{g} \mathrm{MeHg} / \mathrm{g}$ d.w.

contaminated food by $0.5 \%$ of the $P$. reticulata extract provided the best neuroprotection (Fig. 3) since the AChE activity in both the brain and skeletal muscle of fish remained higher than that corresponding measurements in the control fish (50 and 69\%, respectively; Supplementary Table 5). On the other hand, the addition of C. favosus extract to the $\mathrm{MeHg}$-contaminated food suppressed the significant mercurial neurotoxicity in the skeletal muscle (Fig. 3) and levels of AChE activity were $80 \%$ higher than those measured after exposure to $\mathrm{MeHg}$ alone and equivalent to those in the control muscle (Supplementary Table 5).

Expression of sod 1 and $m t 2$ antioxidant and AChEencoding genes in response to $\mathrm{MeHg}$ and plant extractcontaining diets. The effects of 60-day exposure to the different diets on the relative expression of three selected genes were evaluated in the brain, liver and skeletal muscles of the fish (Table 3). Two are involved in the neutralization of the superoxide radical anion and maintenance of the redox balance, sodl, and $m t 2$, encoding the cytoplasmic copper-zinc superoxide dismutase and the second isoform of metallothionein. The third gene was that of AChE, ache.

In the MeHg-fed fish, the $m t 2$ gene was significantly repressed 9-, 4- and 5-fold in the brain, liver, and muscle, respectively (Table 3, Supplementary Table 6). The effect of $\mathrm{MeHg}$ on $m t 2$ gene repression was reversed by both natural extracts, and even an overexpression was observed in the three tested tissues when $P$. reticulata extract was combined with $\mathrm{MeHg}$, with a significant 4-, 24-, and 4fold increased expressions compared to that of the control in the brain, liver, and muscle, respectively. The addition of $C$. favosus extract to food spiked with $\mathrm{MeHg}$ caused a significant 14-fold increase in expression of the $m t 2$ gene.

The exposure to $\mathrm{MeHg}$ triggered a significant 14- and 2.5 -fold downregulation of the sodl gene expression in the liver and muscle, respectively. Supplementation of the $\mathrm{MeHg}$-spiked food with either one of the natural extracts restored the level of sodl gene expression to a level that was similar to that of the control in the liver and muscle.

In the muscle, exposure to $\mathrm{MeHg}$ triggered a significant 5-fold downregulation of ache gene. The supplementation of MeHg-spiked food with either one of the natural extracts reversed the repression, resulting in a level of expression of ache gene similar to the control. Whereas the $\mathrm{MeHg}$ exposure did not induce a differential ache gene expression in the brain, supplementation of the MeHg-spiked food with $P$. reticulata and $C$. favosus extracts triggered a 
Table 3. Relative genes' expression ${ }^{\mathrm{a}}$ reported in the brain, the liver and the muscle of zebrafish fed with different diets during 60 days. Results are presented as mean $\pm \operatorname{SEM}(n=5)$

\begin{tabular}{|c|c|c|c|c|c|c|}
\hline $\begin{array}{l}\text { Tissue } \\
\text { and gene }\end{array}$ & Control & P. reticulata ${ }^{\mathrm{b}}$ & P. reticulata $+\mathrm{MeHg}^{\mathrm{c}}$ & C. favosus ${ }^{\mathrm{b}}$ & C. favosus $+\mathrm{MeHg}^{\mathrm{c}}$ & $\mathrm{MeHg}^{\mathrm{d}}$ \\
\hline \multicolumn{7}{|l|}{ Brain } \\
\hline sod1 & $(6.9 \pm 0.5) \times 10^{-10}$ & $(6.0 \pm 1.0) \times 10^{-1 x}$ & $(131 \pm 26) \times 10^{-1} *$ & $(14 \pm 5) \times 10^{-1}$ & $(150 \pm 50) \times 10^{-1} *$ & $(190 \pm 170) \times 10^{-1} *$ \\
\hline ache & $(4.2 \pm 1.5) \times 10^{-1}$ & $(3.9 \pm 2.1) \times 10^{-1}$ & $(58 \pm 12) \times 10^{-1} *$ & $(6.8 \pm 1.4) \times 10^{-10}$ & $(19 \pm 5) \times 10^{-1} *^{,}$ & $(3.4 \pm 0.5) \times 10^{-1}$ \\
\hline \multicolumn{7}{|l|}{ Liver } \\
\hline$m+2$ & $10 \pm 0.3^{\infty}$ & $6 \pm 0.8^{\alpha}$ & $240 \pm 70^{*, \alpha}$ & $13 \pm 4.2^{\alpha}$ & $140 \pm 80^{*, \alpha}$ & $2.4 \pm 0.05^{*}$ \\
\hline sod1 & $24 \pm 18^{\alpha}$ & $16 \pm 9^{a}$ & $18 \pm 11^{\alpha}$ & $20 \pm 6^{a}$ & $29 \pm 17^{\alpha}$ & $1.7 \pm 0.3^{*}$ \\
\hline \multicolumn{7}{|l|}{ Muscle } \\
\hline$m t 2$ & $1 \pm 0.4^{\infty}$ & $7.7 \pm 4.7^{\alpha}$ & $4 \pm 0.2^{*, \infty}$ & $1.9 \pm 0.9^{\alpha}$ & $1.1 \pm 0.1^{\square}$ & $0.2 \pm 0.1^{*}$ \\
\hline sodl & $(4.9 \pm 0.8) \times 10^{-1 x}$ & $(9.0 \pm 3.1) \times 10^{-10}$ & $(9 \pm 2.9) \times 10^{-1 \varnothing}$ & $(18 \pm 11) \times 10^{-10}$ & $(2.2 \pm 0.5) \times 10^{-1}$ & $(2 \pm 0.3) \times 10^{-1} *$ \\
\hline ache & $1 \pm 0.4^{\alpha}$ & $2 \pm 0.1^{\alpha}$ & $2 \pm 0.2^{\infty}$ & $2 \pm 0.3^{\alpha}$ & $0.9 \pm 0.07^{\alpha}$ & $0.2 \pm 0.07^{*}$ \\
\hline
\end{tabular}

${ }^{\mathrm{a}} \mathrm{Gene}$ expression was calculated using the $2^{\Lambda \mathrm{Ct}}$ method and the $\beta$-actin as reference gene.

${ }^{\mathrm{b}}$ Food supplemented by $0.5 \%$ of either the plant extract of $P$. reticulata or that of $C$. favosus.

${ }^{c}$ Food containing $0.5 \%$ of either the plant extract of $P$. reticulata or that of $C$. favosus and $13.5 \mu \mathrm{g} \mathrm{MeHg} / \mathrm{g}$ (d.w.).

${ }^{\mathrm{d}}$ Fish fed with MeHg contaminated food (13.5 $\mu \mathrm{g} \mathrm{MeHg} / \mathrm{g}$ d.w.).

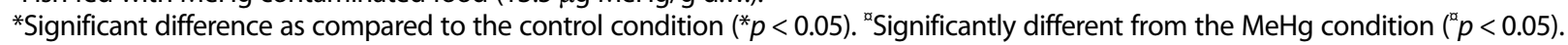

significant 14- and 5-fold increased expression of those genes, respectively.

\section{DISCUSSION}

The protective action of both extracts has been demonstrated, for the first time, in yeast exposed to $\mathrm{CdCl}_{2}$. The $\Delta y c f 1$ mutant strain of $S$. cerevisiae clearly demonstrated a hypersensitivity to cadmium exposure because the wildtype yeast strain resists up to $100 \mu \mathrm{M} \mathrm{Cd}$ (II) (28) and up to $500 \mu \mathrm{M}$ of $\mathrm{Cd}(\mathrm{II})$ when it overexpresses the YCF1 gene (29), whereas in the present study, the half-maximal (50\%) inhibitory concentration (IC50)50 for the $\Delta y c f 1$ mutant was $20 \mu \mathrm{M} \mathrm{CdCl}_{2}$. Thus, considering the hypersensitivity to $\mathrm{CdCl}_{2}$, the results obtained in the $\Delta y c f 1$ mutant clearly demonstrated the potential protective effects of both extracts on the antioxidant defense system of the yeast. In addition, the results more precisely indicate the ability of these extracts to reverse the onset of the expression of genes involved in response to oxidative stress and genotoxic damage triggered by the exposure to $\mathrm{CdCl}_{2}$. Furthermore, this was a successful first step in the selection process of putative metal-protective extracts because challenging yeast with a combination of $\mathrm{CdCl}_{2}$ and extracts saves time and spares animal lives. Finally, the yeast allowed the selection of a single dose of each extract to be used in zebrafish feed contaminated by $\mathrm{MeHg}$.

The results presented here clearly highlight that the mercury-generated oxidative stress, exemplified by increased levels of lipid peroxidation, could be suppressed by food supplementation with $0.5 \%$ of either plant extracts. In addition, the levels could even be maintained at significantly lower levels than the basal levels. Previous studies have demonstrated the in vivo protective potential of plant extracts against metal-generated lipid peroxidation $(13,14$, $16,30,31)$. These results are in line with those observed in our study on the in vivo antioxidant property of both plant extracts. Indeed, while $\mathrm{MeHg}$ downregulated the $m t 2$ gene, both extracts suppressed the effect of mercury, and the $P$. reticulata extract even upregulation of the gene in the brain, liver, and muscle. These observations indicate that these extracts likely stimulated the antioxidant response, paralleling the complete reversal of the lipid peroxidation levels induced by $\mathrm{MeHg}$ in the brain and liver. In agreement with this idea, both extracts reversed the MeHg-promoted sodl gene repression in the liver and muscle.

The analysis of the phytochemical composition of the plant extracts used in our experiment identified the main fraction in both aqueous extracts are phenolic compounds $(5,6)$. These compounds are particularly known to be primary antioxidants, acting as free radical scavengers (32) that delay or inhibit the propagation of lipid peroxidation (33-35). Therefore, they might be responsible for the observed neutralization of the $\mathrm{MeHg}$-generated oxidative stress. The in vitro antioxidant effect of phenolic compounds extracted from garlic (Allium sativum L.) against lipid peroxidation has been shown on a homogenate of the rat kidney exposed to cisplatin (36). Additionally, the antilipid peroxidation property of polyphenolic compounds extracted from the bark and stem of Mahonia leschenaultia takeda (Berberidaceae) and five medicinal plants of the Colombian Amazonia has also been reported $(37,38)$. Therefore, these results corroborate the hypothesis that the anti-lipid peroxidation activity of the aqueous extracts observed in our experiment could be mediated by their rich phenolic compound content. 
Additionally, in the present study, the in vivo neuroprotective potential of the two aqueous extracts against mercurial neurotoxicity was highlighted, as evidenced by reversal of the MeHg-induced $\mathrm{AChE}$ enzyme inhibition. Furthermore, this finding was supported by the suppression of MeHg-induced ache gene repression in the muscle, and the upregulation of this gene was triggered in the brain by both extracts. The neuroprotective potential of the plant extracts against mercury-induced AChE inhibition has also been observed in rats (39) and the lobster cockroach Nauphoeta cinerea (13).

The concentration of accumulated $\mathrm{Hg}$ in the brain and muscle was not modified by treatment with $P$. reticulata and C. favosus extracts. However, a 2-fold reduction of the $\mathrm{Hg}$ concentration in the liver was observed after treatment with $P$. reticulata extract compared to animals exposed only to $\mathrm{MeHg}$ and could explain the protective effects of the extract. The brains and muscles of animals treated with the extract accumulated as much $\mathrm{Hg}$ as those of animals only exposed to MeHg did. Therefore, the weaker Hg concentration in the liver of extract-treated animals than control animals was not due to the modification of the properties of the intestinal barrier or chelation of $\mathrm{MeHg}$ by some compounds of the extract in the luminal part of the intestine. Rather, this observed effect was likely due to an increase in the detoxification capabilities of the liver. Indeed, when food was spiked with $\mathrm{MeHg}$, the expression of the $m t 2$ gene in the liver was 100 -fold greater in presence of $P$. reticulata extract than in its absence, and this was paralleled by a 2-fold drop in Hg concentration. Such an increase in $\mathrm{MeHg}$ sequestering capability might augment the cellular depuration of this toxic compound through dedicated $\mathrm{ABC}$ transporters. In agreement with this observation, the antioxidant $\mathrm{Nrf2}$ transcription factor was shown to be stimulated in murine hepatocytes and it was necessary to explain the protective effect against $\mathrm{MeHg}$ generated by vegetal isothiocyanate. This eventually decreased the intracellular $\mathrm{MeHg}$ concentration mediated by the increased concentrations of MRP1 and MRP2 ABC transporters (40-42). Similarly, the Skn1 transcription factor, which is the homolog of the mammalian Nrf2 in Caenorhabditis elegans, is involved in the resistance of this nematode worm to $\mathrm{MeHg}$, and the ethanolic extract of the guarana plant was shown to protect $s k n$-1-mutated nematodes against $\mathrm{MeHg}$ (43). MeHg also increased Nrf2 activation by the downregulation of the Fyn kinase. This is induced by MeHg-mediated activation of Akt kinase, which inhibits glycogen synthase kinase $3 \beta$, leading to the retention of the Fyn kinase in the cytoplasm, and in turn, suppresses the Fyn-mediated exclusion of Nrf2 from the nucleus (44). This second intracellular signaling pathway is stimulated by $\mathrm{MeHg}$ and might explain why even with the presence of $\mathrm{MeHg}$ in the food, the protective effect of both extracts against lipid peroxidation in the liver was greater than that in the control animals (both neutralization of MeHg-induced radicals by phenolic compounds and $\mathrm{MeHg}$-mediated stimulation of $\mathrm{Nrf2}$ ).

In conclusion, in the present study, we demonstrated the in vivo protective potential of both $P$. reticulata and $C$. favosus aqueous extracts against mercury toxicity. These results are of great interest since the Amazonian riverside populations are still exposed to mercury poisoning through the consumption of Hg-contaminated fish (45). Indeed, these populations exhibit a high level of exposure to mercury with a mean hair mercury level, a bio-indicator of $\mathrm{Hg}$ exposure, which can reach up to $15 \mu \mathrm{g} \mathrm{Hg} / \mathrm{g}$ in the highly exposed populations. This level of mercury represents the highest concentration reported worldwide and is associated with deleterious effects (12). The known effects of mercury exposure include nervous system damage in adults and impaired neurological development in infants and children (17). Hence, the results showing the protective potential of both aqueous extracts against $\mathrm{MeHg}$ in zebrafish could represent an interesting alternative strategy for the Amazonian fish-eating communities to address the impact of chronic exposure to MeHg-contaminated diets. However, the applicability of these findings is based on the assumption that the molecular mechanisms underlying $\mathrm{MeHg}$ toxicity and the protective properties of the extracts are similar in zebrafish and humans.

\section{ACKNOWLEDGMENTS}

This study was funded by the French National Institute for Universe Sciences (INSU), CNRS, through its program EC2CO, the University of West of Pará (UFOPA), and the FAPESPA (the Amazon Research Foundation that promotes science and technology in the state of Pará), the National Council for Scientific and Technological Development $(\mathrm{CNPq})$, and the Agency for the Support and Evaluation of Graduate Education (CAPES) for providing financial support [grant numbers 88881.030.472/2013-01 and 88881.030.467/2013-01].

\section{CONFLICT OF INTEREST} kind.

We declare that we have no conflict of interest of any

Received May 16, 2018; Revised June 21, 2018; Accepted July 20,2018

\section{REFERENCES}

1. Bieski, I.G., Leonti, M., Arnason, J.T., Ferrier, J., Rapinski, M., Violante, I.M., Balogun, S.O., Pereira, J.F., Figueiredo Rde C., Lopes, C.R., da Silva, D.R., Pacini, A., Albuquerque, U.P. and Martins, D.T. (2015) Ethnobotanical study of 
medicinal plants by population of Valley of Juruena Region, Legal Amazon, Mato Grosso, Brazil. J. Ethnopharmacol., 173, 383-423.

2. Breitbach, U.B., Niehues, M., Lopes, N.P., Faria, J.E.Q. and Brandão, M.G.L. (2013) Amazonian Brazilian medicinal plants described by C.F.P. von Martius in the 19th century. $J$. Ethnopharmacol., 147, 180-189.

3. De Medeiros, P.M., Ladio, A.H. and Albuquerque, U.P. (2013) Patterns of medicinal plant use by inhabitants of Brazilian urban and rural areas: a macroscale investigation based on available literature. J. Ethnopharmacol., 150, 729746.

4. Pedrollo, C.T., Kinupp, V.F., Shepard, G., Jr. and Heinrich, M. (2016) Medicinal plants at Rio Jauaperi, Brazilian Amazon: ethnobotanical survey and environmental conservation. J. Ethnopharmacol., 186, 111-124.

5. Moura, V.M., da Silva, W.C., Raposo, J.D., Freitas-de-Sousa, L.A., Dos-Santos, M.C., de Oliveira, R.B. and Veras Mourão, R.H. (2016) The inhibitory potential of the condensed-tannin-rich fraction of Plathymenia reticulata Benth. (Fabaceae) against Bothrops atrox envenomation. J. Ethnopharmacol., 183, 136-142.

6. Pereira da Silva, T., Moura, V.M., Souza, M.C., Santos, V.N., Silva, K.A., Mendes, M.G., Nunez, C.V., Almeida, P.D., Lima, E.S., Mourão, R.H. and Dos-Santos, M.C. (2016) Connarus favosus Planch.: an inhibitor of the hemorrhagic activity of Bothrops atrox venom and a potential antioxidant and antibacterial agent. J. Ethnopharmacol., 183, 166-175.

7. Moura, V.M., Freitas-de-Sousa, L.A., Dos-Santos, M.C., Raposo, J.D., Lima, A.E., de Oliveira, R.B., da Silva, M.N. and Veras Mourão, R.H. (2015) Plants used to treat snakebites in Santarém, western Pará, Brazil: an assessment of their effectiveness in inhibiting hemorrhagic activity induced by Bothrops jararaca venom. J. Ethnopharmacol., 161, 224232.

8. Cavalcanti, D.R. and Albuquerque, U.P. (2013) The "hidden diversity" of medicinal plants in northeastern Brazil: diagnosis and prospects for conservation and biological prospecting. Evid. Based. Complement. Alternat. Med., 2013, 102714.

9. Nascimento, J.M. and Conceição, G.M. (2011) Plantas medicinais e indicações terapêuticas da comunidade Quilombola Olho D'água do Raposo, Caxias, Maranhão, Brasil. Rev. Biol. Farmácia., 6, 138-151.

10. Berzas Nevado, J.J., Rodríguez Martín-Doimeadios, R.C., Guzmán Bernardo, F.J., Jiménez Moreno, M., Herculano, A.M., do Nascimento, J.L. and Crespo-López, M.E. (2010) Mercury in the Tapajós river basin, Brazilian amazon: a review. Environ. Int., 36, 593-608.

11. Passos, C.J.S., Mergler, D., Fillion, M., Lemire, M., Mertens, F., Guimarães, J.R.D. and Philibert, A. (2007) Epidemiologic confirmation that fruit consumption influences mercury exposure in the Brazilian Amazon. Environ. Res., 105, 183193.

12. Passos, C.J.S. and Mergler, D. (2008) Human mercury exposure and adverse health effects in the Amazon: a review. Cad. Saude Publica, 24, S503-S520.

13. Adedara, I.A., Rosemberg, D.B., Souza, D.O., Farombi, E.O., Aschner, M. and Rocha, J.B. (2016) Neuroprotection of luteolin against methylmercury-induced toxicity in lobster cockroach Nauphoeta cinerea. Environ. Toxicol. Pharmacol., 42, 243-251.

14. Ayyathan, D.M., Chandrasekaran, R. and Thiagarajan, K. (2012) Neuroprotective effect of Tagara, an Ayurvedic drug against methyl mercury induced oxidative stress using rat brain mitochondrial fractions. BMC Complement Altern Med., 15, 268.

15. Farina, M., Rocha, J.B. and Aschner, M. (2011) Mechanisms of methylmercury-induced neurotoxicity: evidence from experimental studies. Life Sci., 89, 555-563.

16. Kim, W., Kim, D.W., Yoo, D.Y., Jung, H.Y., Kim, J.W., Kim, D.W., Choi, J.H., Moon, S.M., Yoon, Y.S. and Hwang, I.K. (2015) Antioxidant effects of Dendropanax morbifera Léveille extract in the hippocampus of mercury-exposed rats. BMC Complement Altern Med., 15, 247.

17. Rice, K.M., Walker, E.M. Jr., Wu, M., Gillette, C. and Blough, E.R. (2014) Environmental mercury and its toxic effects. J. Prev. Med. Public. Health, 47, 74-83.

18. Sharma, M.K., Kumar, M. and Kumar, A. (2005) Protection against mercury-induced renal damage in Swiss albino mice by Ocimum sanctum. Environ. Toxicol. Pharmacol., 19, 161167.

19. Li, Z.S., Lu, Y.P., Zhen, R.G., Szczypka, M., Thiele, D.J. and Rea, P.A. (1997) A new pathway for vacuolar cadmium sequestration in Saccharomyces cerevisiae: YCF1-catalyzed transport of bis(glutathionato)cadmium. Proc. Natl. Acad. Sci. U.S.A., 94, 42-47.

20. Endo-Ichikawa, Y., Kohno, H., Tokunaga, R. and Taketani, S. (1995) Induction in the gene RNR3 in Saccharomyces cerevisiae upon exposure to different agents related to carcinogenesis. Biochem. Pharmacol., 50, 1695-1699.

21. Vido, K., Spector, D., Lagniel, G., Lopez, S., Toledano, M.B. and Labarre, J. (2001) A proteome analysis of the cadmium response in Saccharomyces cerevisiae. J. Biol. Chem., 276, 8469-8474.

22. Gonzalez, P., Dominique, Y., Massabuau, J.C., Boudou, A. and Bourdineaud, J.P. (2005) Comparative effects of dietary methylmercury on gene expression in liver, skeletal muscle, and brain of the zebrafish (Danio rerio). Environ. Sci. Technol., 39, 3972-3980.

23. Cambier, S., Bénard, G., Mesmer-Dudons, N., Gonzalez, P., Rossignol, R., Brèthes, D. and Bourdineaud, J.P. (2009) At environmental doses, dietary methylmercury inhibits mitochondrial energy metabolism in skeletal muscles of the zebra fish (Danio rerio). Int. J. Biochem. Cell Biol., 41,791-799.

24. De Oliveira Ribeiro, C.A., Nathalie, M.D., Gonzalez, P., Yannick, D., Bourdineaud, J.P., Boudou, A. and Massabuau, J.C. (2008) Effects of dietary methylmercury on zebrafish skeletal muscle fibres. Environ. Toxicol. Pharmacol., 25, 304-309.

25. Cambier, S., Gonzalez, P., Mesmer-Dudons, N., Brèthes, D., Fujimura, M. and Bourdineaud, J.P. (2012) Effects of dietary methylmercury on the zebrafish brain: histological, mitochondrial, and gene transcription analyses. Biometals, 25, 165-180.

26. Oakes, K.D., McMaster, M.E. and Van Der Kraak, G.J. (2003) Utility of the TBARS assay in detecting oxidative stress in white sucker (Catostomus commersoni) popula- 
tions exposed to pulp mill effluent. Aquat. Toxicol., 63, 447463.

27. Ellman, G.L., Courtney, K., Andres, V.J.R. and FeatherStone, R.M. (1961) A new and rapid colorimetric determination of AChE activity. Biochem. Pharmacol., 7, 88-95.

28. Wemmie, J.A., Szczypka, M.S., Thiele, D.J. and MoyeRowley, W.S. (1994) Cadmium tolerance mediated by the yeast AP-1 protein requires the presence of an ATP-binding cassette transporter-encoding gene, YCF1. J. Biol. Chem., 269, 32592-32597.

29. Szczypka, M.S., Wemmie, J.A., Moye-Rowley, W.S. and Thiele, D.J. (1994) A yeast metal resistance protein similar to human cystic fibrosis transmembrane conductance regulator (CFTR) and multidrug resistance-associated protein. $J$. Biol. Chem., 269, 22853-22857.

30. Farina, M., Franco, J.L., Ribas, C.M., Meotti, F.C., Missau, F.C., Pizzolatti, M.G., Dafre, A.L. and Santos, A.R. (2005) Protective effects of Polygala paniculata extract against methylmercury-induced neurotoxicity in mice. J. Pharm. Pharmacol., 57, 1503-1508.

31. Tunali-Akbay, T., Sener, G., Salvarli, H., Sehirli, O. and Yarat, A. (2007) Protective effects of Ginkgo biloba extract against mercury(II)-induced cardiovascular oxidative damage in rats. Phytother. Res., 21, 26-31.

32. Kang, M.H. and Kim, B.H. (2018) Oral wound healing effects of acai berry water extracts in rat oral mucosa. Toxicol. Res., 34, 97-102.

33. Figueroa, L.A., Navarro, L.B., Vera, M.P. and Petricevich, V.L. (2014) Antioxidant activity, total phenolic and flavonoid contents, and cytotoxicity evaluation of Bougainvillea xbuttiana. Int. J. Pharm. Pharm. Sci., 6, 497-502.

34. Karagöz, A., Artun, F.T., Özcan, G., Melikoğlu, G., Anıl, S., Kültür, S. and Sütlüpınar, N. (2015) In vitro evaluation of antioxidant activity of some plant methanol extracts. Biotechnol. Biotechnol. Equip., 29, 1184-1189.

35. Shahidi, F. and Ambigaipalan, P. (2015) Phenolics and polyphenolics in foods, beverages and spices: antioxidant activity and health effects - a review. J. Funct. Foods, 18, 820897.

36. Oboh, G., Akinyemi, A.J. and Ademiluyi, A.O. (2013) Inhibitory effect of phenolic extract from garlic on angioten- sin-1 converting enzyme and cisplatin induced lipid peroxidation - In Vitro. Int. J. Biomed. Sci., 9, 98-106.

37. Adithya, E.S., Lakshmi, M.S., Christabel, P.H. and Sasikumar, J.M. (2013) In vitro antioxidant, anti-lipid peroxidation activities and HPLC analysis of methanol extracts from bark and stem of Mahonia leschenaultia takeda. Asian J. Plant. Sci. Res., 3,116-126.

38. Lizcano, L.J., Viloria-Bernal, M., Vicente, F., Berrueta, L.A., Gallo, B., Martínez-Cañamero, M., Ruiz-Larrea, M.B. and Ruiz-Sanz, J.I. (2012) Lipid oxidation inhibitory effects and phenolic composition of aqueous extracts from medicinal plants of Colombian Amazonia. Int. J. Mol. Sci., 13, 54545467.

39. Christinal, J. and Sumathi, T. (2014) Chronic administration of methylmercury alters cognitive and mitochondrial dysfunction in rat cerebellum - a novel approach to therapy for Minamata disease by a medhya rasayana drug Bacopa monniera. Int. J. Pharm. Bio. Sci., 5, 1183-1194.

40. Toyama, T., Shinkai, Y., Yasutake, A., Uchida, K., Yamamoto, M. and Kumagai, Y. (2011) Isothiocyanates reduce mercury accumulation via an Nrf2-dependent mechanism during exposure of mice to methylmercury. Environ. Health Perspect., 119, 1117-1122.

41. Kumagai, Y., Kanda, H., Shinkai, Y. and Toyama, T. (2013) The role of the Keap1/Nrf2 pathway in the cellular response to methylmercury. Oxid. Med. Cell. Longev., 2013, 848279.

42. Gum, S.I. and Cho, M.K. (2013) Recent updates on acetaminophen hepatotoxicity: the role of $\mathrm{Nrf} 2$ in hepatoprotection. Toxicol. Res., 29, 165-172.

43. Arantes, L.P., Peres, T.V., Chen, P., Caito, S.W., Aschner, M. and Soares, F.A. (2016) Guarana (Paullinia cupana Mart.) attenuates methylmercury-induced toxicity in Caenorhabditis elegans. Toxicol. Res., 5, 1629-1638.

44. Culbreth, M., Zhang, Z. and Aschner, M. (2017) Methylmercury augments Nrf2 activity by downregulation of the Src family kinase Fyn. Neurotoxicology, 62, 200-206.

45. Bourdineaud, J.P., Durrieu, G., Sarrazin, S.L., da Silva, W.C., Mourão, R.H. and de Oliveira, R.B. (2015) Mercurial exposure of residents of Santarém and Oriximiná cities (Pará, Brazil) through fish consumption. Environ. Sci. Pollut. Res. Int., 22, 12150-12161. 